\title{
Influence of adiposity, insulin resistance, and intrahepatic triglyceride content on insulin kinetics
}

\author{
Gordon I. Smith, ${ }^{1}$ David C. Polidori, ${ }^{2}$ Mihoko Yoshino, ${ }^{1}$ Monica L. Kearney, ${ }^{1}$ Bruce W. Patterson, ${ }^{1}$ Bettina Mittendorfer, \\ and Samuel Klein ${ }^{1}$ \\ 'Center for Human Nutrition, Washington University School of Medicine, St. Louis, Missouri, USA. 'Janssen Research \& Development, San Diego, California, USA.
}

BACKCROUND. Insulin is a key regulator of metabolic function. The effects of excess adiposity, insulin resistance, and hepatic steatosis on the complex integration of insulin secretion and hepatic and extrahepatic tissue extraction are not clear.

\begin{abstract}
METHODS. A hyperinsulinemic-euglycemic clamp and a 3-hour oral glucose tolerance test were performed to evaluate insulin sensitivity and insulin kinetics after glucose ingestion in 3 groups: (a) lean subjects with normal intrahepatic triglyceride (IHTG) and glucose tolerance (lean-NL; $n=14$ ), (b) obese subjects with normal IHTC and glucose tolerance (obese-NL; $n=24$ ), and (c) obese subjects with nonalcoholic fatty liver disease (NAFLD) and prediabetes (obese-NAFLD; $\boldsymbol{n}=22$ ).

RESULTS. Insulin sensitivity progressively decreased and insulin secretion progressively increased from the lean-NL to the obese-NL to the obese-NAFLD groups. Fractional hepatic insulin extraction progressively decreased from the lean-NL to the obese-NL to the obese-NAFLD groups, whereas total hepatic insulin extraction (molar amount removed) was greater in the obese-NL and obese-NAFLD subjects than in the lean-NL subjects. Insulin appearance in the systemic circulation and extrahepatic insulin extraction progressively increased from the lean-NL to the obese-NL to the obese-NAFLD groups. Total hepatic insulin extraction plateaued at high rates of insulin delivery, whereas the relationship between systemic insulin appearance and total extrahepatic extraction was linear.
\end{abstract}

CONCLUSION. Hyperinsulinemia after glucose ingestion in obese-NL and obese-NAFLD is due to an increase in insulin secretion, without a decrease in total hepatic or extrahepatic insulin extraction. However, the liver's maximum capacity to remove insulin is limited because of a saturable extraction process. The increase in insulin delivery to the liver and extrahepatic tissues in obese-NAFLD is unable to compensate for the increase in insulin resistance, resulting in impaired glucose homeostasis.

TRIAL REGISTRATION. ClinicalTrials.gov NCT02706262.

FUNDING. NIH grants DK56341 (Nutrition Obesity Research Center), DK052574 (Digestive Disease Research Center), RR024992 (Clinical and Translational Science Award), and T32 DK007120 (a T32 Ruth L. Kirschstein National Research Service Award); the American Diabetes Foundation (1-18-ICTS-119); Janssen Research \& Development; and the Pershing Square Foundation.

\section{Introduction}

Obesity is associated with nonalcoholic fatty liver disease (NAFLD), multiorgan insulin resistance, and hyperinsulinemia, which are major risk factors for both type 2 diabetes and coronary heart disease (1-4). Although hyperinsulinemia and insulin resistance are likely involved in the pathogenesis of NAFLD (5), excess intrahepatic triglyceride (IHTG) content could also contribute to hyperinsulinemia and insulin resistance. The liver is important in regulating systemic plasma insulin concentrations, because it is the major site for insulin

Conflict of interest: SK is a shareholder of Aspire Bariatrics, receives research funding from Janssen Pharmaceuticals Inc., and serves on the scientific advisory boards for Danone and Merck Sharp \& Dohme Corp. DCP is an employee of Janssen Research \& Development. Copyright: () 2020, American Society for Clinical Investigation.

Submitted: January 27, 2020; Accepted: March 11, 2020; Published: May 18, 2020

Reference information: J Clin Invest. 2020;130(6):3305-3314.

https://doi.org/10.1172/JCl136756. clearance; in individuals who are lean and healthy, a large portion $(-50 \%)$ of the insulin delivered to the liver is cleared during firstpass transit, and an additional $20 \%$ is cleared through subsequent passes $(6,7)$. The remaining $30 \%$ of insulin secreted by the pancreas is removed by extrahepatic organs, primarily the kidneys and skeletal muscle $(6,8)$. Increased insulin secretion and impaired hepatic insulin clearance in individuals with NAFLD could contribute to insulin resistance by chronic exposure of insulin-sensitive tissues to large amounts of insulin, which can downregulate insulin receptor binding affinity and insulin receptor numbers (9-12). Even 24 hours of an experimentally induced increase in plasma insulin concentration causes hepatic and skeletal muscle insulin resistance (13), and a single dose of a pharmacological agent that decreases insulin secretion lowers 24-hour plasma glucose and insulin concentrations and improves oral glucose tolerance (14) in healthy, lean adults. However, the relationship between IHTG content and insulin kinetics is not clear because of conflicting data from different studies that found 
Table 1. Body composition and metabolic characteristics of the study subjects

\begin{tabular}{|c|c|c|c|}
\hline & Lean-NL $(n=14)$ & Obese-NL $(n=24)$ & Obese-NAFLD $(n=22)$ \\
\hline Body weight, kg & $65 \pm 2$ & $106 \pm 4^{A}$ & $116 \pm 4^{\mathrm{A}}$ \\
\hline $\mathrm{BMI}, \mathrm{kg} / \mathrm{m}^{2}$ & $23 \pm 1$ & $38 \pm 1^{A}$ & $40 \pm 1^{A}$ \\
\hline $\mathrm{FFM}, \mathrm{kg}$ & $46 \pm 2$ & $55 \pm 2^{A}$ & $59 \pm 2^{A}$ \\
\hline Body fat, \% & $28 \pm 2$ & $47 \pm 1^{A}$ & $48 \pm 1^{A}$ \\
\hline IHTG content, \% & $1.6 \pm 0.1$ & $2.4 \pm 0.2$ & $21.0 \pm 1.4^{\mathrm{A}, \mathrm{B}}$ \\
\hline HbA1c, \% & $5.0 \pm 0.1$ & $5.1 \pm 0.1$ & $5.7 \pm 0.1^{A, B}$ \\
\hline Fasting glucose, $\mathrm{mmol} / \mathrm{L}$ & $4.7 \pm 0.1$ & $4.9 \pm 0.1$ & $5.6 \pm 0.1^{A, B}$ \\
\hline Fasting insulin, pmol/L & $36 \pm 3$ & $84 \pm 7^{A}$ & $196 \pm 23^{A, B}$ \\
\hline Fasting C-peptide, pmol/L & $487 \pm 34$ & $826 \pm 34^{A}$ & $1,491 \pm 94^{A, B}$ \\
\hline OGTT 2-hour glucose, $\mathrm{mmol} / \mathrm{L}$ & $5.3 \pm 0.3$ & $6.0 \pm 0.2$ & $9.4 \pm 0.3^{\mathrm{A}, \mathrm{B}}$ \\
\hline $\mathrm{HISI}, 100 /(\mu \mathrm{mol} / \mathrm{kg} \mathrm{FFM} / \mathrm{min} \times \mu \mathrm{U} / \mathrm{mL})$ & $1.13 \pm 0.12$ & $0.53 \pm 0.04^{A}$ & $0.30 \pm 0.03^{A, B}$ \\
\hline Glucose Rd/insulin, (nmol/kg FFM/min)/(pmol/L) & $89 \pm 6$ & $56 \pm 5^{A}$ & $30 \pm 2^{A, B}$ \\
\hline
\end{tabular}

Values represent the mean \pm SEM. HISI, hepatic insulin sensitivity index. A 1-way ANOVA with post hoc testing where appropriate was used to identify significant mean differences among groups. ${ }^{A} P<0.05$, value significantly different from the corresponding value in the lean-NL group; ${ }^{\mathrm{B}} P<0.05$, value significantly different from the corresponding value in the obese-NL group. plasma glucose concentrations, plasma glucose 2 hours after glucose ingestion, and hemoglobin A1c (HbA1c) were higher in the obese-NAFLD group than in the obese-NL and lean-NL groups, with no differences between the obese-NL and lean-NL groups (Table 1). Fasting plasma insulin and C-peptide concentrations increased progressively from the lean-NL to the obese-NL to the obese-NAFLD groups (Table 1). Both hepatic insulin sensitivity (assessed as the reciprocal of the product of basal endogenous glucose production rate and basal plasma insulin concentration) and muscle insulin sensitivity (assessed as the glucose disposal rate relative to the plasma insulin concentration during the HECP) decreased progressively from the leanNL to the obese-NL to the obese-NAFLD groups (Table 1).

insulin secretion was either increased or the same and insulin clearance was either decreased or the same in individuals with NAFLD compared with those with normal IHTG content (15-18). The reason for the differences between studies could be related to differences in characteristics of the study subjects and the methods used to assess IHTG content and insulin metabolism.

The purpose of the present study was to provide a comprehensive evaluation of the complex interrelationship among obesity, insulin resistance, hepatic steatosis, and insulin kinetics. A hyperinsulinemic-euglycemic clamp procedure (HECP), in conjunction with stable isotopically labeled glucose tracer infusion, and a 3-hour oral glucose tolerance test (OGTT) were used to evaluate insulin sensitivity and insulin kinetics in 3 cohorts of individuals who differed in adiposity, insulin sensitivity, and IHTG content: (a) lean with normal IHTG content and normal fasting glucose and oral glucose tolerance (lean-NL), (b) obese with normal IHTG content and normal fasting glucose and oral glucose tolerance (obese-NL), and (c) obese with high IHTG content and evidence of abnormal glucose metabolism (impaired fasting glucose or oral glucose tolerance) (obese-NAFLD). We used a recently developed modeling approach $(15,19)$ in conjunction with C-peptide deconvolution to assess different aspects of insulin kinetics in response to glucose ingestion, including the insulin secretion rate and hepatic, extrahepatic, and whole-body insulin plasma clearance and tissue extraction rates. A better understanding of the interrelationships among adiposity, IHTG content, insulin sensitivity, and insulin kinetics can provide new insights into the mechanisms that regulate glucose homeostasis in individuals with obesity and those with obesity and NAFLD.

\section{Results}

Body composition and metabolic characteristics. The obese-NL and obese-NAFLD groups were matched with regard to BMI and body fat percentage, but IHTG content was 9-fold greater in the obese-NAFLD group than in the obese-NL group, with no difference in IHTG content between the lean-NL and obese-NL groups (Table 1). Fasting
Plasma glucose, insulin, and C-peptide responses to glucose ingestion. Both plasma glucose concentrations and plasma glucose AUC after glucose ingestion were greater in the obese-NAFLD group than in the obese-NL and lean-NL groups, which were not significantly different from each other (Figure 1, A and B). Plasma insulin and C-peptide concentrations and AUC after glucose ingestion increased progressively from the lean-NL to the obese-NL to the obese-NAFLD groups (Figure 1, C-F). The plasma insulin concenthat in the obese-NL group and 3.5 times greater than in the leanNL group, whereas the plasma C-peptide concentration AUC was only $50 \%$ greater in the obese-NAFLD group than in the obese-NL group and 2-fold greater than in the lean-NL group.

Insulin kinetics. The kinetics model accurately described the insulin data from both the OGTT and HECP (average normalized root mean square error: $6.3 \% \pm 3.5 \%$ ) (Supplemental Figure 1 ; supplemental material available online with this article; https:// doi.org/10.1172/JCI136756DS1). The amount of insulin delivered to the liver comprises both insulin secreted by $\beta$ cells and insulin that passes through the liver into the systemic circulation and is recycled back to the liver. Both the insulin secretion rate (ISR) and the rate of insulin recycled back to the liver during the 3-hour OGTT increased progressively from the lean-NL to the obese-NL to the obese-NAFLD groups (Figure 2A). Fractional hepatic insulin extraction (i.e., the fraction of insulin delivered to the liver that is removed by the liver) decreased progressively from the lean-NL to the obese-NL to the obese-NAFLD groups and was significantly lower in the obese-NAFLD group than in the obese-NL and leanNL groups (Figure 2B). However, the rate of total hepatic insulin extraction (i.e., the molar amount of insulin removed from plasma by the liver per minute) progressively increased from the lean-NL to the obese-NL to the obese-NAFLD groups and was greater in both the obese-NAFLD and obese-NL groups than in the lean-NL group, with no difference observed between the obese-NAFLD and obese-NL groups (Figure 2C). Although the fractional extraction tration AUC in the obese-NAFLD group was 2-fold greater than 
A

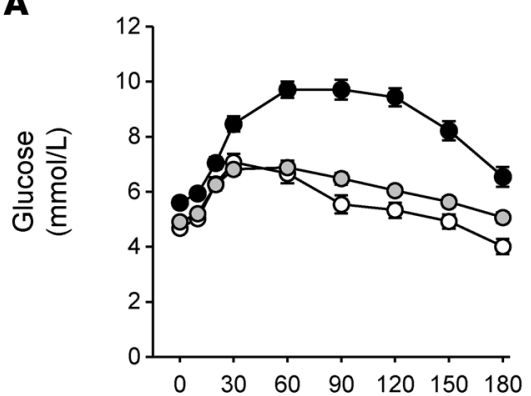

C

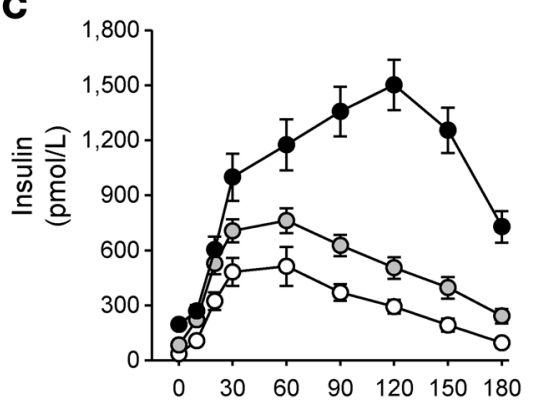

E

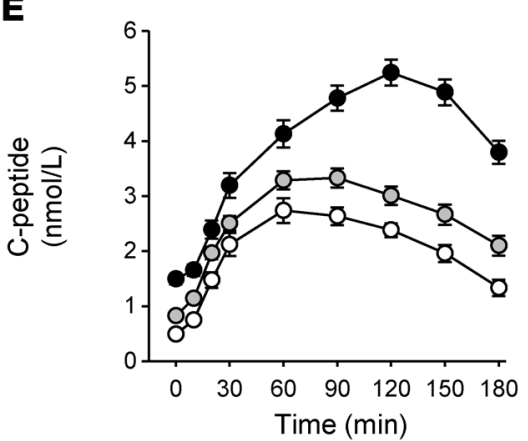

B


$\mathbf{F}$

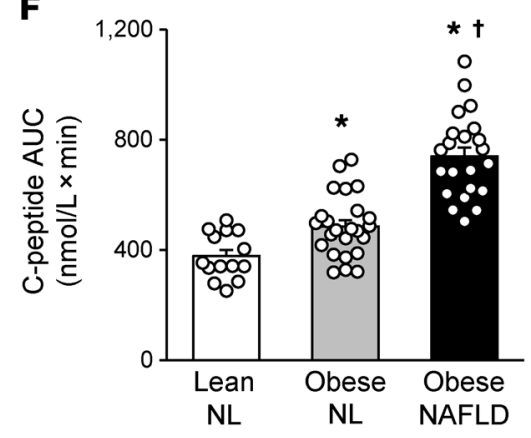

Figure 1. Plasma glucose, insulin, and C-peptide responses to glucose ingestion. Plasma glucose, insulin, and C-peptide concentrations before and over a 3-hour period after ingesting a 75-g glucose drink (A, C, and E), and plasma glucose, insulin, and C-peptide 3 -hour concentration AUC values (B, $\mathbf{D}$, and $\mathbf{F}$ ) in the lean-normal (NL), obese-NL, and obese-NAFLD groups. White, gray, and black circles in $\mathbf{A}, \mathbf{C}$, and $\mathbf{E}$ represent the lean-NL $(n=14)$, obese-NL $(n=24)$, and obese-NAFLD $(n=22)$ groups, respectively. Values in B, D, and $\mathbf{F}$ represent the mean \pm SEM. $P$ values were determined by 1-way ANOVA with post hoc testing to identify significant mean differences between groups when appropriate. ${ }^{*} P<0.05$, value significantly different from the lean-NL value; ${ }^{\dagger} P<0.05$, value significantly different from the obese-NL value.

observed in the obese-NL group, with considerable variability in the rate of hepatic insulin extraction at any given rate of insulin delivery (Figure 2G). In contrast, the total extrahepatic insulin extraction rate increased linearly with increasing rates of insulin delivery into the systemic circulation (i.e., total posthepatic insulin appearance in plasma) (Figure 2H).

Interrelationships among insulin sensitivity, plasma insulin concentration, IHTG content, and insulin kinetics. The whole-body insulin clearance rate (i.e., volume of plasma cleared of insulin per minute) during the OGTT was positively correlated with insulin sensitivity (assessed as the glucose rate of disposal relative to the plasma insulin concentration [glucose $\mathrm{Rd} / \mathrm{I}$ ] during the HECP) (Figure 3A), whereas the whole-body insulin extraction rate (i.e., the molar amount of

of insulin by extrahepatic tissues (i.e., the fraction of insulin delivered to extrahepatic tissues that is removed) was not different in the lean-NL $(34 \% \pm 2 \%)$, obese-NL $(28 \% \pm 3 \%)$, or obese-NAFLD $(30 \% \pm 2 \%)(P=0.60)$ group, the rate of total extrahepatic insulin extraction (i.e., the molar amount of insulin removed by extrahepatic tissues per minute) progressively increased from the lean-NL to the obese-NL to the obese-NAFLD groups and was more than double the rate in the obese-NAFLD group than in the obeseNL group (Figure 2D). The rate of total (whole-body) insulin extraction increased progressively from the lean-NL to the obeseNL to the obese-NAFLD groups because of increases in both total hepatic and extrahepatic insulin extraction rates (Figure 2E). The liver accounted for approximately $70 \%$ of whole-body insulin extraction in the lean-NL and obese-NL groups, but only approximately $50 \%$ in the obese-NAFLD group; conversely, extrahepatic insulin extraction increased from approximately $30 \%$ of wholebody insulin extraction in the lean-NL and obese-NL groups to approximately $50 \%$ in the obese-NAFLD group (Figure $2 \mathrm{~F}$ ). The relationship between the rate of insulin delivered to the liver (i.e., ISR and posthepatic insulin that is not removed by extrahepatic tissues and is recycled back to the liver) and the rate of total hepatic insulin extraction demonstrated a saturable process that began to plateau with the increase in hepatic insulin delivery rates insulin removed per minute) was negatively correlated with muscle insulin sensitivity (Figure 3B). The rate of whole-body insulin clearance was negatively correlated with the plasma insulin AUC during the OGTT (Figure 3C), whereas the whole-body insulin extraction rate was positively correlated with the plasma insulin AUC and was best described by a saturable, Michaelis-Menten relationship (ref. 20 and Figure 3D), presumably driven by the saturability of hepatic insulin extraction. We observed no significant correlation between either the fractional hepatic insulin extraction rate or the total hepatic insulin extraction rate and IHTG content in the obese-NAFLD group (Supplemental Figure 2).

Indices of $\beta$ cell function. The ISR during the OGTT was inversely correlated with muscle insulin sensitivity, and the ISR increased as muscle insulin sensitivity decreased in a curvilinear fashion (Figure $4 \mathrm{~A})$. The $\beta$ cell function index (i.e., the incremental ISR in relation to muscle insulin sensitivity), which provides a measure of insulin secretion by $\beta$ cells in relation to insulin sensitivity, decreased progressively from the lean-NL to the obese-NL to the obese-NAFLD groups and was significantly lower in the obese-NAFLD group than in the lean-NL and obese-NL groups (Figure 4B). Therefore, the high ISR in the obese-NL group adequately compensated for the decrease in insulin sensitivity needed to maintain normal oral glucose tolerance. However, even the very high ISR in the obese- 
A

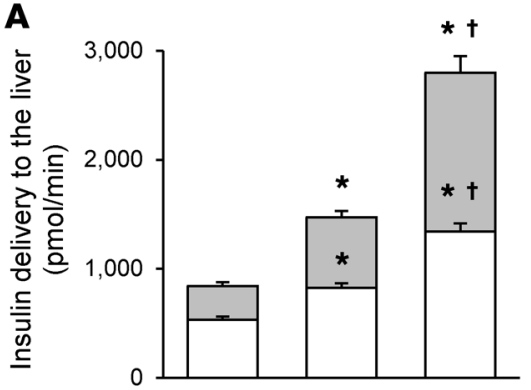

C



E

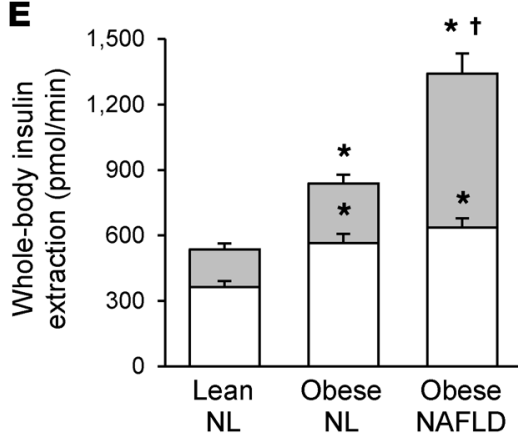

G

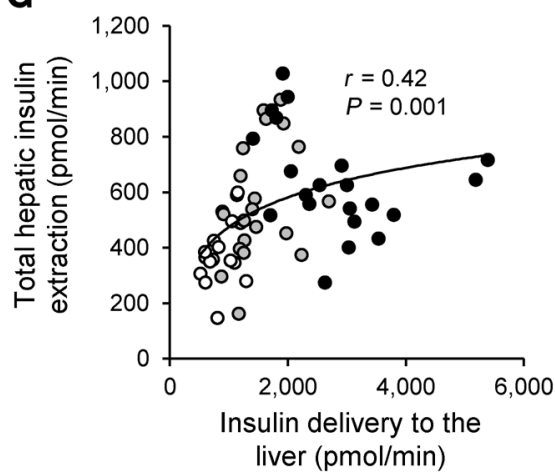

B



D



$\mathbf{F}$

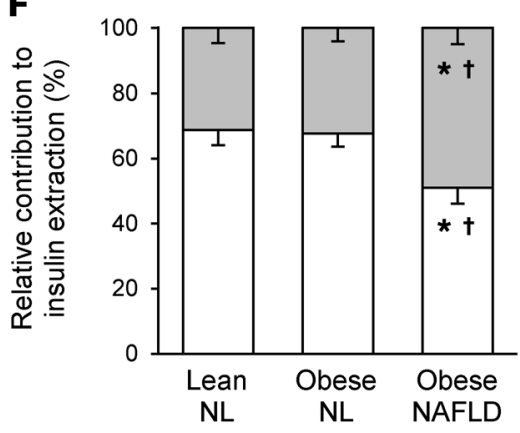

H



Figure 2. Insulin kinetics after glucose ingestion. Rate of total insulin delivered to the liver, comprising the rate of insulin secreted from $\beta$ cells (white bars) and the rate of insulin recycled from the systemic circulation back to the liver (gray bars) (A), fractional hepatic insulin extraction (B), rate of total hepatic insulin extraction (C), rate of total extrahepatic insulin extraction (D), absolute contribution of hepatic (white bars) and extrahepatic (gray bars) insulin extraction to the total rate of whole-body insulin extraction (E), and relative contribution of hepatic (white bars) and extrahepatic (gray bars) extraction to the total rate of whole-body insulin extraction (F) in the lean-NL $(n=14)$, obese-NL $(n=23)$, and obese-NAFLD ( $n$ $=21$ ) groups. Values represent the mean \pm SEM and indicate the averages for 3 hours after glucose ingestion. A 1-way ANCOVA with race and sex as covariates and post hoc testing where appropriate were used to identify significant mean differences between groups. ${ }^{*} P<0.05$, value significantly different from the lean-NL value; ${ }^{\dagger} P<0.05$, value significantly different from the obese-NL value. Relationship between insulin delivery to the liver and the rate of total hepatic insulin extraction (C) and relationship between the insulin delivery rate into the systemic circulation and the rate of total extrahepatic insulin extraction $(\mathbf{H})$ in lean-NL (white circles; $n=14$ ), obese-NL (gray circles; $n=$ 23 ), and obese-NAFLD (black circles; $n=21$ ) participants. Logarithmic and linear regression analyses were performed to determine the line of best fit to the data in $\mathbf{G}$ and $\mathbf{H}$, respectively.

of hepatic and muscle insulin sensitivity measured during the HECP, these groups represented a progressive deterioration in insulin sensitivity from the lean-NL to the obese$\mathrm{NL}$ to the obese-NAFLD groups. We used a recently developed modeling approach (15, 19) and C-peptide deconvolution to provide a comprehensive analysis of insulin kinetics in response to glucose ingestion, including insulin secretion by $\beta$ cells and hepatic, extrahepatic, and whole-body insulin plasma clearance and tissue extraction rates. The major findings from our study are: (a) the ISR in response to glucose ingestion progressively increased from the lean-NL to the obese-NL to the obese-NAFLD groups, but $\beta$

NAFLD group was not adequate to compensate for the further decrease in insulin sensitivity in the obese-NAFLD group, resulting in abnormal glucose tolerance (Figure 1A).

\section{Discussion}

We conducted an OGTT and a HECP in 3 carefully characterized cohorts of participants who were either lean with normal glucose tolerance and normal IHTG content, obese with normal glucose tolerance and normal IHTG content, or obese with prediabetes and NAFLD to help dissect the effects of adiposity, insulin resistance, and hepatic steatosis on insulin kinetics. Based on the assessment cell function, assessed as the increase in ISR in relation to insulin sensitivity, was lower in the obese-NAFLD group than in the leanNL and obese-NL groups; (b) hepatic steatosis does not impair the rate of hepatic insulin extraction (molar amount of insulin removed from plasma per unit of time), and total hepatic insulin extraction rates were greater in the obese-NL and obese-NAFLD groups than in the lean-NL group, but were not different between the obese-NAFLD and obese-NL groups; (c) the rate of total extrahepatic insulin extraction progressively increased from the lean-NL to the obese-NL to the obese-NAFLD groups; (d) the total hepatic insulin extraction rate plateaued when hepatic insulin delivery 



Figure 3. Relationships among insulin sensitivity and insulin concentration after glucose ingestion and whole-body insulin clearance and extraction rates. Relationships among whole-body insulin clearance and extraction rates assessed for 3 hours after ingestion of a 75 -g glucose drink and muscle insulin sensitivity, calculated as the glucose Rd (in nmol/kg FFM/ $\mathrm{min}$ ) divided by the plasma insulin (I) concentration (in pmol/L) during a HECP (A and B), and the plasma insulin concentration AUC (C and D). White, gray, and black circles represent participants in the lean-NL ( $n$ $=14$ ), obese-NL ( $n=24$ in A and C and $n=23$ in B and D), and obese-NAFLD ( $n=22$ in A and $\mathbf{C}$ and $n=21$ in B and D) groups, respectively. Logarithmic regression analysis was used to determine the lines of best fit to the data in A-C, with Michaelis-Menten kinetics used to describe the line of best fit in $\mathbf{D}$.

of insulin to the liver (prehepatic insulin) from lean-NL to obese-NL to obese-NAFLD groups. The liver's ability to increase the rate of insulin extraction when insulin delivery to the liver was increased, as in the obese-NL and obese-NAFLD groups, was limited, presumably because of a saturable hepatic insulin transport system

(from newly secreted and recycled insulin) was high, whereas the total extrahepatic insulin extraction rate increased linearly with increasing delivery of insulin into the systemic circulation; and (e) the whole-body insulin clearance rate (volume of plasma cleared of insulin) was positively correlated, whereas the whole-body insulin extraction rate (the molar amount of insulin removed by all tissues) was negatively correlated with muscle insulin sensitivity. Our data demonstrate that the greater increase in plasma insulin concentrations in response to an oral glucose challenge in the obese-NL and obese-NAFLD groups compared with that observed in the lean-NL group is due to an increase in insulin secretion, not a reduction in total insulin extraction by the liver or extrahepatic tissues. However, the liver's capacity to remove insulin is a saturable process that reaches maximum capacity when hepatic insulin delivery is high, which occurred after glucose ingestion in our obese-NL and obese-NAFLD subjects. In contrast, extrahepatic insulin extraction after glucose ingestion increased linearly with increases in insulin delivery into the systemic circulation. Nonetheless, the marked increases in hepatic and extrahepatic insulin delivery and extraction in the obese-NAFLD group were not adequate to compensate for the decrease in insulin sensitivity, resulting in impaired glucose homeostasis.

Systemic plasma insulin concentration is determined by the rate of insulin secretion by $\beta$ cells and the rate of insulin removal by the liver and extrahepatic tissues. The results from our study provide an integrated assessment of insulin kinetics in response to an oral glucose challenge, including rates of insulin secretion, hepatic and extrahepatic insulin extraction, and recycling of posthepatic insulin back to the liver in distinct cohorts of individuals who differed in adiposity, IHTG content, and hepatic and muscle insulin sensitivity (Figure 5). The data demonstrate that both insulin secreted by the pancreas and insulin recycled from the systemic circulation progressively increased the total delivery
(21-23). Therefore, an increase in the delivery of insulin to the liver was associated with a decrease in fractional hepatic insulin extraction, and more insulin passed through the liver into the systemic circulation. Most of the insulin that entered the systemic circulation (posthepatic insulin) was recycled back to the liver, but a progressively increasing amount of insulin was removed by extrahepatic tissues (primarily the kidneys and skeletal muscle) $(6,8)$ in the lean-NL, obese-NL, and obese-NAFLD groups. In all groups, more than $99 \%$ of insulin secreted by $\beta$ cells was removed by hepatic and extrahepatic tissues during the 180-minute OGTT. However, small differences between the rate of insulin secretion and removal among the 3 groups resulted in marked differences in plasma insulin concentration at the 180-minute time point $(60$ $\pm 22,158 \pm 38$, and $532 \pm 80 \mathrm{pmol} / \mathrm{L}$ in the lean-NL, obese-NL, and obese-NALFD groups, respectively) (Figure 1C). These results demonstrate that the major factor responsible for hyperinsulinemia in individuals with obesity who have insulin resistance and NAFLD is $\beta$ cell hypersecretion in conjunction with a saturable insulin extraction process in the liver.

Although there was a large range in IHTG content in the obese-NAFLD group, we found no correlation between either fractional hepatic insulin extraction or the rate of total hepatic insulin extraction and the severity of steatosis. In addition, the total hepatic insulin extraction rate was not significantly different between the obese-NAFLD and obese-NL groups. These results challenge the notion that NAFLD per se impairs hepatic insulin extraction. However, we also found considerable variability in the hepatic insulin extraction rate at any given rate of insulin delivery to the liver in the obese-NL and obese-NAFLD groups. The reasons for the heterogeneity in the rates of total hepatic insulin extraction are not clear but could be related to individual subject variability in some of the assumed values the kinetic model uses, such as hepatic blood flow and C-peptide 



Figure 4. Indices of $\boldsymbol{\beta}$ cell function. (A) Relationship between muscle insulin sensitivity, calculated as the glucose Rd (in $\mathrm{nmol} / \mathrm{kg} \mathrm{FFM/min)} \mathrm{divided}$ by the plasma insulin (I) concentration (in pmol/L) during a HECP, and the mean insulin secretion rate, assessed for 3 hours after ingestion of a $75-\mathrm{g}$ glucose drink in lean-NL (white circles; $n=14$ ), obese-NL (gray circles; $n$ $=24$ ), and obese-NAFLD (black circles; $n=22$ ) participants. Logarithmic regression analysis was used to determine the line of best fit to the data. (B) $\beta$ Cell function index, calculated as the product of the incremental insulin secretion rate (in $\mathrm{nmol} \times \mathrm{min}$ ) for 3 hours after glucose ingestion $\left(\triangle \mathrm{ISR}_{0-180}\right)$ and muscle insulin sensitivity. Values represent the mean \pm SEM. A 1-way ANCOVA with race and sex as covariates and post hoc testing where appropriate were used to identify significant mean differences between groups. ${ }^{*} P<0.05$, value significantly different from the lean-NL value; ${ }^{\dagger} P<0.05$, value significantly different from the obese-NL value.

kinetics, differences in the expression of insulin receptors, and differences in the content of intrahepatic proteins involved in insulin degradation, namely hepatic carcinoembryonic antigen-related cell adhesion molecule 1 (CEACAM1) and insulin-degrading enzyme (IDE) (24).

In individuals with normal glucose tolerance, there is a hyperbolic relationship between insulin sensitivity and the increase in plasma insulin concentration in response to an oral or intravenous glucose challenge; the product of these 2 variables is known as the disposition index (DI) (25-27). Accordingly, DI values are maintained when a decrease or increase in insulin sensitivity is compensated by a corresponding increase or decrease, respectively, in the plasma insulin response to a glucose load (28-30). However, the prevailing plasma insulin concentration is a function of both the rate of insulin secretion and the rate of insulin removal. Therefore, the DI concept implies that $\beta$ cells and the liver are somehow able to sense changes in whole-body insulin sensitivity and adjust the rate of insulin secretion and removal as needed to prevent hypoglycemia, while increasing circulating insulin to compensate for insulin resistance. The data from our study suggest that insulin secretion drives this process, whereas hepatic insulin extraction is likely a passive function of insulin delivery that becomes saturated at high insulin delivery rates. Despite the very high ISR and plasma insulin concentrations after glucose ingestion in the obese-NAFLD group, postprandial plasma glucose concentrations were much higher in the obese-NAFLD group than in the obese$\mathrm{NL}$ and lean-NL groups. Therefore, the increased $\beta$ cell response and increase in plasma insulin concentrations in the obese-NAFLD group were unable to compensate for the increase in insulin resistance, which is consistent with the observed lower $\beta$ cell function index (i.e., incremental insulin secretion in relation to insulin sensitivity) in the obese-NAFLD group than in the lean-NL and obese-NL groups.

Our study was unable to determine whether the increased ISRs in the obese-NL and obese-NAFLD groups were a cause or a consequence of insulin resistance, or possibly both. The increased ISRs could be due to a proposed $\beta$ cell compensatory response to insulin resistance (31), which is consistent with the inverse correlation between the ISR and whole-body insulin sensitivity observed among subjects in our entire cohort. However, the mechanism responsible for the ability of the $\beta$ cell to "sense" insulin resistance in other tissues has not been identified. Increased insulin secretion can also be caused by intrinsic $\beta$ cell hyperreactivity to substrate, hormonal and neural stimuli, and even environmental pollutants $(32,33)$. In addition, the normal feedback suppression of insulin secretion by circulating insulin is blunted in obese individuals (34). Accordingly, hyperinsulinemia in individuals with obesity could lead to a "vicious insulin cycle," in which increased insulin secretion causes insulin resistance, which in turn stimulates increased insulin secretion. The high rate of insulin secretion and plasma insulin concentrations can have adverse long-term clinical consequences, because a high ISR is a risk factor for developing type 2 diabetes $(29,32)$. These findings suggest that the most effective approach for preventing prediabetes and type 2 diabetes in obese individuals should include interventions that decrease insulin secretion and plasma concentration $(35,36)$.

Several limitations of our study should be considered. First, differences in insulin secretion and clearance rates have been reported among different racial/ethnic groups (37-39), so the results from our study, which primarily included White $(65 \%)$ and African American (27\%) participants, might not apply to other racial/ethnic populations. In an effort to reduce the potential confounding effect of race, we included race as a covariate in our statistical analyses. In addition, we performed additional analyses that evaluated the data from the White and African American participants separately. All significant differences between lean-NL, obese-NL, and obese-NAFLD groups and significant correlations between outcome measures were maintained when evaluating White participants only. The same pattern of differences in outcomes between groups and the correlations between outcomes were maintained for the African American participants, but some of these assessments did not achieve statistical significance because of inadequate sample size in this subgroup. Second, the model used to assess insulin kinetics includes estimated values for C-peptide kinetic parameters and hepatic blood flow that are based on standard estimates that do not fully account for interindividual variability and are assumed to be the same during the 

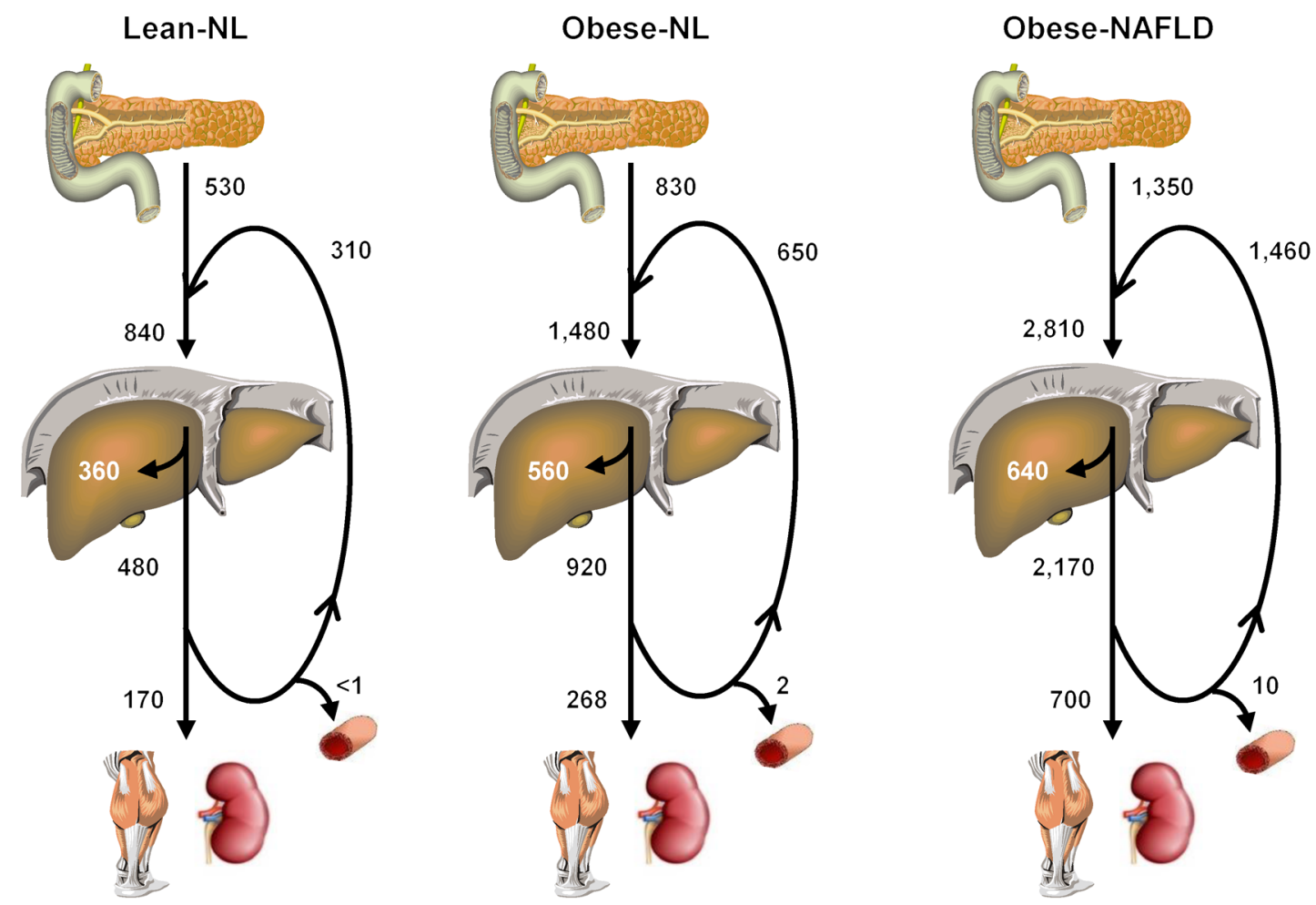

Figure 5. Integrated summary of insulin kinetics after glucose ingestion. Values represent the mean rates (in pmol/min) for $\beta$ cell insulin secretion, tissue insulin extraction, and insulin accumulation in the systemic circulation, assessed for 3 hours after ingestion of a $75-\mathrm{g}$ glucose drink. Insulin secretion by the pancreas into the portal circulation increased progressively from the lean-NL to the obese-NL to the obese-NAFLD groups. In addition, a large portion of insulin that entered the portal circulation was not immediately removed by the liver and extrahepatic tissues and was recycled back to the liver via the portal vein and hepatic artery, so the total amount of insulin delivered to the liver (newly secreted and recycled insulin) also increased progressively from the lean-NL to the obese-NL to the obese-NAFLD groups. Although the fractional hepatic extraction of delivered insulin progressively decreased, the rate of total hepatic insulin extraction progressively increased from the lean-NL to the obese-NL to the obese-NAFLD groups. However, the rate of hepatic insulin extraction plateaued when the delivery of insulin to the liver was high, as in the obese-NL and obese-NAFLD groups, because of a saturable hepatic insulin transport system. Most of the insulin that passes through the liver and enters the systemic circulation is recycled back to the liver, and a progressively increasing amount of insulin was removed by extrahepatic tissues (primarily the kidneys and skeletal muscle) in subjects in the lean-NL, obese-NL, and obese-NAFLD groups. A small portion of insulin that entered the systemic circulation (posthepatic insulin) was not removed by 180 minutes after glucose ingestion and was responsible for the increase in plasma insulin concentration above baseline at the 180-minute time point.

OGTT and the HECP. Third, our study is a cross-sectional analysis of weight-stable participants, so we cannot exclude the possibility that changes in insulin kinetics occur over time or in response to changes in diet or body weight.

In summary, the large increase in plasma insulin concentrations in response to an oral glucose challenge that is commonly observed in individuals with obesity and further exacerbated in individuals with obesity who have NAFLD and greater insulin resistance was driven by increased insulin secretion, without an intrinsic defect in hepatic or extrahepatic insulin extraction. Therefore, the progressive decrease in whole-body insulin clearance rates (volume of plasma cleared of insulin per unit of time) from lean-NL to obese-NL to obese-NAFLD was probably a consequence, rather than a cause, of hyperinsulinemia. The rate of insulin extraction by the liver, but not by extrahepatic tissues, became saturable when the postprandial delivery of insulin to the liver was high. In individuals with severe insulin resistance, the increased delivery of insulin to the liver and extrahepatic tissues was unable to compensate for the decrease in insulin sensitivity, resulting in impaired glucose homeostasis.

\section{Methods}

Subjects. A total of 60 men and women participated in this study (see Supplemental Figure 3 for the flow chart of study subjects). Subjects were recruited using the Volunteers for Health database at Washington University School of Medicine and by local postings between April 2016 and November 2018. All procedures for this study were conducted in the Clinical Translational Research Unit (CTRU) and Center for Clinical Imaging Research (CCIR) at Washington University School of Medicine. Potential subjects completed an initial evaluation that included a medical history and physical examination, standard blood tests, a 3-hour OGTT and an assessment of body composition including IHTG content. Subjects were enrolled if they met the criteria for inclusion in 1 of 3 groups: (a) lean-NL individuals, defined as having a BMI of 18.5 to $24.9 \mathrm{~kg} / \mathrm{m}^{2}$ and normal fasting plasma glucose $(<100 \mathrm{mg} / \mathrm{dL})$, oral glucose tolerance (2-hour glucose $<140 \mathrm{mg} / \mathrm{dL}$ ), and IHTG content ( $\leq 5 \%)(n=$ 14; age: $36 \pm 2 \mathrm{yr}$; sex: 7 men and 7 women; race: 9 Whites, 1 African American, and 4 Asians); (b) obese-NL individuals, defined as having a BMI of 30.0 to $49.9 \mathrm{~kg} / \mathrm{m}^{2}$ and normal fasting plasma glucose, oral glucose tolerance, and IHTG content $(n=24$; age: $39 \pm 2 \mathrm{yr}$; 
sex: 3 men, 21 women; race: 13 Whites and 11 African Americans); and (c) obese-NAFLD individuals, defined as having a BMI of 30.0 to $49.9 \mathrm{~kg} / \mathrm{m}^{2}$, impaired fasting glucose or oral glucose tolerance, and high IHTG content $(\geq 10 \%)(n=22$; age: $42 \pm 2 \mathrm{yr}$; sex: 6 men and 16 women; race: 17 Whites, 4 African Americans, and 1 Pacific Islander). None of the subjects had evidence of diabetes, serious illnesses other than NAFLD, was taking medications that could interfere with insulin action or secretion, consumed excessive amounts of alcohol (>14 drinks/week for women and >21 drinks/week for men), or smoked tobacco products.

Body composition analyses. Total body fat and fat-free mass (FFM) were determined by using dual-energy x-ray absorptiometry (Lunar iDXA, GE Healthcare), and IHTG content was determined by MRI (3.0-T superconducting magnet; Siemens) (5).

OGTT. Subjects were admitted to the CTRU at Washington University School of Medicine at 0700 hours after subjects fasted for approximately 11 hours overnight at home. An intravenous catheter was inserted into an antecubital or hand vein for serial blood sampling. Plasma glucose, insulin, and C-peptide concentrations were determined 15, 10, and 5 minutes before and 10, 20, 30, 60, 90, 120, 150 , and 180 minutes after consuming a $75-\mathrm{g}$ glucose beverage. The average of the 3 baseline samples (i.e., $-15,-10$, and -5 minutes before consuming the 75 -g glucose beverage) was used as the $t=0$ glucose, insulin, and plasma C-peptide concentrations.

HECP. Subjects were admitted to the CTRU at 1800 hours for approximately 48 hours. Participants were given standard meals containing one-third of their estimated energy requirements (40) upon admission (day 0) and at 0700 hours, 1300 hours, and 1900 hours on day 1 . The HECP was performed on day 2, after subjects fasted overnight. At 0700 hours, a primed $(8.0 \mu \mathrm{mol} / \mathrm{kg})$, continuous $(0.08 \mu \mathrm{mol} /$ $\mathrm{kg} / \mathrm{min}$ ) infusion of $\left[\mathrm{U}^{13} \mathrm{C}\right]$ glucose (Cambridge Isotope Laboratories Inc.) was started. After the infusion of glucose tracer for 3.5 hours (basal period), insulin was infused at a rate of $50 \mathrm{mU} / \mathrm{m}^{2} / \mathrm{min}$ (initiated with a 2-step priming dose of $200 \mathrm{mU} / \mathrm{m}^{2} / \mathrm{min}$ for 5 minutes followed by $100 \mathrm{mU} / \mathrm{m}^{2} / \mathrm{min}$ for 5 minutes), and euglycemia $(\sim 100 \mathrm{mg} / \mathrm{dL})$ was maintained by variable-rate infusion of a $20 \%$ dextrose solution that was enriched to approximately $1 \%$ with $\left[\mathrm{U}-{ }^{13} \mathrm{C}\right]$ glucose. The infusion of $\left[\mathrm{U}-{ }^{13} \mathrm{C}\right]$ glucose was stopped during insulin infusion because of the expected decrease in hepatic glucose production. Blood samples were obtained before beginning the glucose tracer infusion and every 6 to 7 minutes during the last 20 minutes of the basal and insulin infusion stages to determine glucose, C-peptide, and insulin concentrations and glucose kinetics.

Sample analyses and calculations. Blood samples were collected in chilled tubes containing EDTA or heparin and placed in ice. Plasma was separated by centrifugation within 30 minutes of collection and then stored at $-80^{\circ} \mathrm{C}$ until final analyses. The plasma glucose concentration was determined using the glucose oxidase method (YSI Inc.), and plasma insulin and C-peptide concentrations were determined using electrochemiluminescence assays (Elecsys 2010, Roche Diagnostics). The plasma glucose tracer-to-tracee ratio was determined by gas-chromatography/mass-spectrometry as described previously (41).

Plasma glucose, insulin, and C-peptide concentration AUCs during the OGTT were calculated using the trapezoidal method (42). Hepatic insulin sensitivity was calculated as the reciprocal of the product of the basal endogenous glucose production rate (in $\mu \mathrm{mol} / \mathrm{kg} \mathrm{FFM} /$ $\mathrm{min}$ ) and the basal plasma insulin concentration (in $\mu \mathrm{U} / \mathrm{mL}$ ) (1). Total glucose Rd during insulin infusion was assumed to be equal to the sum of the endogenous glucose rate of appearance into the bloodstream and the rate of infused glucose during the last 20 minutes of the HECP (1). An index of muscle insulin sensitivity was calculated as the glucose Rd expressed per kilogram of FFM divided by the plasma insulin concentration (glucose $\mathrm{Rd} / \mathrm{I}$ ) during the final 20 minutes of the HECP. Insulin secretion rates were calculated using $\mathrm{C}$-peptide deconvolution (43). Insulin secretion in relationship to insulin sensitivity was used to provide an index of $\beta$ cell function and calculated as the product of the incremental AUC in the ISR above time 0 from 0 to 180 minutes of the OGTT, and insulin sensitivity was assessed during the HECP $\left(\triangle \mathrm{ISR}_{0-180}\right.$ $\times$ glucose $\mathrm{Rd} / \mathrm{I})$.

The whole-body insulin clearance rate (i.e., volume of plasma cleared of insulin per minute) was calculated using a 1-compartment model for plasma insulin: (AUC ISR/AUC I) $-\mathrm{V} \times\left(\mathrm{I}_{180}-\mathrm{I}_{0}\right) / A U C \mathrm{I}$, where $\mathrm{V}$ is the distribution volume for insulin estimated as $141 \mathrm{~mL} /$ $\mathrm{kg}(19)$, and $\mathrm{I}_{0}$ and $\mathrm{I}_{180}$ are the plasma insulin concentrations at time 0 (baseline) and 180 minutes, respectively, during the OGTT. A recently developed mathematical modeling approach that involves the use of plasma insulin concentration and ISR data from both the OGTT and HECP $(15,19)$ was used to provide a comprehensive assessment of the kinetics of hepatic and extrahepatic insulin removal from plasma during the OGTT. In this model, hepatic insulin clearance for each subject was modeled using either a linear or saturable model, and the model that provided the better fit was used for that subject. In addition, extrahepatic insulin clearance was assumed to be linear; this assumption was confirmed by testing a saturable model for extrahepatic insulin clearance, and finding a linear model provided the best fit of the data for all subjects. The following measurements of insulin kinetics were determined: (a) fractional hepatic insulin extraction (i.e., the fraction of insulin delivered to the liver that is removed by the liver); (b) total hepatic insulin extraction rate (i.e., molar amount of insulin removed from plasma by the liver per minute); (c) rate of insulin recycled from the systemic circulation back to the liver (i.e., insulin that passes through the liver into the systemic circulation that is not removed by extrahepatic tissues and is recycled back to the liver); (d) rate of extrahepatic insulin extraction (i.e., molar amount of insulin removed from plasma by extrahepatic tissues per unit of time); and (e) whole-body insulin extraction rate (i.e., sum of the hepatic and extrahepatic insulin extraction rates).

Statistics. A 1-way ANOVA was used to compare characteristics of subjects in the lean-NL, obese-NL, and obese-NAFLD groups. Between-group differences in the insulin secretion rate, clearance rate, total extraction rate, and fractional extraction were assessed using ANCOVA with race and sex as covariates. Where appropriate, post hoc analyses were used to locate significant mean differences. Modeled and measured plasma insulin concentration profiles were compared using the normalized root mean square error, as previously described $(15,19)$. The significance of the relationships among outcome measures were evaluated using either linear or nonlinear regression. Relationships that involved IHTG content were analyzed separately for subjects with normal IHTG content (lean-NL and obese-NL groups) and high IHTG content (obese-NAFLD group), because there was no continuum in IHTG content according to the study's design. The relationship between the whole-body insulin extraction rate and the plasma insulin concentration AUC during the OGTT was assessed using Michaelis-Menten kinetics to determine whether the rate of 
insulin extraction could be explained by saturable, receptor-mediated insulin uptake. Statistical significance was defined as a $P$ value under 0.05. Statistical analyses were performed using SPSS, version 25 (IBM). Data are reported as the mean \pm SEM. On the basis of the interindividual variability in fractional hepatic insulin extraction we previously reported (15), we estimated that 15 subjects in each group would be needed to detect between-group differences in fractional hepatic insulin extraction rates of $20 \%$ using a 2 -sided test with a $\beta$ value of 0.9 and an $\alpha$ value of 0.05 . These computations were performed using $\mathrm{G}^{*}$ Power, version 3.1.9.2 (44).

Study approval. All subjects provided written informed consent before participating in this study, which was approved by the Human Research Protection Office of Washington University School of Medicine.

\section{Author contributions}

GIS, MY, and MLK conducted the studies. DCP performed the insulin kinetics modeling. BWP supervised the sample analyses. GIS, DCP, BWP, BM, and SK analyzed the data, performed the statistical analyses, and wrote the manuscript. SK designed and supervised the studies and obtained funding for the work. SK is the guarantor of this work and, as such, had full access to all the data in the study and takes responsibility for the integrity of the data and the accuracy of the data analysis. All authors critically reviewed and edited the manuscript.

\section{Acknowledgments}

The authors thank the staff of the Center for Human Nutrition, the Clinical and Translational Research Unit, and the Center for Clinical Imaging Research for their help in performing this study, and the study subjects for their participation. This study was funded by NIH grants DK56341 (Nutrition Obesity Research Center), DK052574 (Digestive Disease Research Center), RR024992 (Clinical and Translational Science Award), and T32 DK007120 (a T32 Ruth L. Kirschstein National Research Service Award); the American Diabetes Foundation (1-18-ICTS-119); Janssen Research $\&$ Development; and the Pershing Square Foundation.

Address correspondence to: Samuel Klein, Center for Human Nutrition, Washington University School of Medicine, 660 South Euclid Avenue, Campus Box 8031, St. Louis, Missouri 63110, USA. Phone: 314.362.8708; Email: sklein@wustl.edu.

MLK's present address is: Department of Kinesiology, Nutrition and Recreation, Southeast Missouri State University, Missouri, USA.
1. Korenblat KM, Fabbrini E, Mohammed BS, Klein S. Liver, muscle, and adipose tissue insulin action is directly related to intrahepatic triglyceride content in obese subjects. Gastroenterology. 2008;134(5):1369-1375.

2. Fabbrini E, et al. Intrahepatic fat, not visceral fat, is linked with metabolic complications of obesity. Proc Natl Acad Sci USA. 2009;106(36):15430-15435.

3. Fabbrini E, Sullivan S, Klein S. Obesity and nonalcoholic fatty liver disease: biochemical, metabolic, and clinical implications. Hepatology. 2010;51(2):679-689.

4. Magkos F, Fabbrini E, Mohammed BS, Patterson BW, Klein S. Increased whole-body adiposity without a concomitant increase in liver fat is not associated with augmented metabolic dysfunction. Obesity (Silver Spring). 2010;18(8):1510-1515.

5 . Smith GI, et al. Insulin resistance drives hepatic de novo lipogenesis in nonalcoholic fatty liver disease. JClin Invest. 2020;130(3):1453-1460.

6. Ferrannini E, Wahren J, Faber OK, Felig P, Binder C, DeFronzo RA. Splanchnic and renal metabolism of insulin in human subjects: a dose-response study. Am J Physiol. 1983;244(6):E517-E527.

7. Peiris AN, Mueller RA, Smith GA, Struve MF, Kissebah AH. Splanchnic insulin metabolism in obesity. Influence of body fat distribution. JClin Invest. 1986;78(6):1648-1657.

8. Duckworth WC, Bennett RG, Hamel FG. Insulin degradation: progress and potential. Endocr Rev. 1998;19(5):608-624.

9. Burén J, Liu HX, Lauritz J, Eriksson JW. High glucose and insulin in combination cause insulin receptor substrate- 1 and -2 depletion and protein kinase $\mathrm{B}$ desensitisation in primary cultured rat adipocytes: possible implications for insulin resistance in type 2 diabetes. Eur JEndocrinol. 2003;148(1):157-167.

10. Kobayashi M, Olefsky JM. Effect of experimental hyperinsulinemia on insulin binding and glucose transport in isolated rat adipocytes. Am J Physiol. 1978;235(1):E53-E62.

11. Blackard WG, Guzelian PS, Small ME. Down regulation of insulin receptors in primary cultures of adult rat hepatocytes in monolayer. Endocrinology. 1978;103(2):548-553.

12. Kalant N, Ozaki S, Maekubo H, Mitmaker B, Cohen-Khallas M. Down-regulation of insulin binding by human and rat hepatocytes in primary culture: the possible role of insulin internalization and degradation. Endocrinology. 1984;114(1):37-43.

13. Iozzo P, et al. Physiological hyperinsulinemia impairs insulin-stimulated glycogen synthase activity and glycogen synthesis. Am J Physiol Endocrinol Metab. 2001;280(5):E712-E719.

14. Zdravkovic M, Kruse M, Rost KL, Møss J, Kecskes A, Dyrberg T. The effects of NN414, a SUR1/ Kir6. 2 selective potassium channel opener, in healthy male subjects. J Clin Pharmacol. 2005;45(7):763-772.

15. Utzschneider KM, Kahn SE, Polidori DC. Hepatic insulin extraction in NAFLD is related to insulin resistance rather than liver fat content. JClin Endocrinol Metab. 2019;104(5):1855-1865.

16. Bril F, et al. Relationship between disease severity, hyperinsulinemia, and impaired insulin clearance in patients with nonalcoholic steatohepatitis. Hepatology. 2014;59(6):2178-2187.

17. Kotronen A, Juurinen L, Tiikkainen M, Vehkavaara S, Yki-Järvinen H. Increased liver fat, impaired insulin clearance, and hepatic and adipose tissue insulin resistance in type 2 diabetes. Gastroenterology. 2008;135(1):122-130.

18. Bril F, et al. Metabolic and histological implications of intrahepatic triglyceride content in nonalcoholic fatty liver disease. Hepatology. 2017;65(4):1132-1144.

19. Polidori DC, Bergman RN, Chung ST, Sumner AE. Hepatic and extrahepatic insulin clearance are differentially regulated: results from a novel model-based analysis of intravenous glucose tolerance data. Diabetes. 2016;65(6):1556-1564.

20. Michaelis L, Menten ML, Johnson KA, Goody RS. The original Michaelis constant: translation of the 1913 Michaelis-Menten paper. Biochemistry. 2011;50(39):8264-8269.

21. Donner DB. Receptor- and non-receptor-mediated uptake and degradation of insulin by hepatocytes. Biochem J. 1982;208(1):211-219.

22. Mondon CE, Olefsky JM, Dolkas CB, Reaven GM. Removal of insulin by perfused rat liver: effect of concentration. Metab Clin Exp. 1975;24(2):153-160.

23. Eaton RP, Allen RC, Schade DS. Hepatic removal of insulin in normal man: dose response to endogenous insulin secretion. J Clin Endocrinol Metab. 1983;56(6):1294-1300.

24. Najjar SM, Perdomo G. Hepatic insulin clearance: mechanism and physiology. Physiology (Bethesda). 2019;34(3):198-215.

25. Kahn SE, et al. Quantification of the relationship between insulin sensitivity and beta-cell function in human subjects. Evidence for a hyperbolic function. Diabetes. 1993;42(11):1663-1672.

26. Buchanan TA, et al. Response of pancreatic beta-cells to improved insulin sensitivity in women at high risk for type 2 diabetes. Diabetes. 2000;49(5):782-788.

27. Bergman RN, Phillips LS, Cobelli C. Physiologic evaluation of factors controlling glucose tolerance in man: measurement of insulin sensitivity and beta-cell glucose sensitivity from the response to intravenous glucose. J Clin Invest. 1981;68(6):1456-1467.

28. DeFronzo RA, Tripathy D, Abdul-Ghani M, Musi $\mathrm{N}$, Gastaldelli A. The disposition index does not reflect $\beta$-cell function in IGT subjects treated with pioglitazone. JClin Endocrinol Metab. 2014;99(10):3774-3781.

29. Magkos F, et al. Effects of moderate and subse- 


\section{CLINICAL MEDICINE}

quent progressive weight loss on metabolic function and adipose tissue biology in humans with obesity. Cell Metab. 2016;23(4):591-601.

30. Fabbrini E, et al. Metabolically normal obese people are protected from adverse effects following weight gain. JClin Invest. 2015;125(2):787-795.

31. Bergman RN, Ader M, Huecking K, Van Citters G. Accurate assessment of beta-cell function: the hyperbolic correction. Diabetes. 2002;51 Suppl 1:S212-S220.

32. Tricò D, Natali A, Arslanian S, Mari A, Ferrannini E. Identification, pathophysiology, and clinical implications of primary insulin hypersecretion in nondiabetic adults and adolescents. JCI Insight . 2018;3(24):124912.

33. Corkey BE. Diabetes: have we got it all wrong? Insulin hypersecretion and food additives: cause of obesity and diabetes? Diabetes Care. 2012;35(12):2432-2437.

34. Elahi D, et al. Feedback inhibition of insulin secretion by insulin: relation to the hyperinsulinemia of obesity. N Engl JMed. 1982;306(20):1196-1202.

35. Greenwood RH, Mahler RF, Hales CN. Improvement in insulin secretion in diabetes after diazoxide. Lancet. 1976;1(7957):444-447.

36. Boland BB, et al. Pancreatic $\beta$-cell rest replenishes insulin secretory capacity and attenuates diabetes in an extreme model of obese type 2 diabetes. Diabetes. 2019;68(1):131-140.

37. Goran MI, Bergman RN, Cruz ML, Watanabe R. Insulin resistance and associated compensatory responses in african-american and Hispanic children. Diabetes Care. 2002;25(12):2184-2190.

38. Piccinini F, Polidori DC, Gower BA, Bergman RN. Hepatic but not extrahepatic insulin clearance is lower in African American than in European American women. Diabetes. 2017;66(10):2564-2570.

39. Piccinini F, Polidori DC, Gower BA, Fernandez JR, Bergman RN. Dissection of hepatic versus extra-hepatic insulin clearance: ethnic differences in childhood. Diabetes Obes Metab.
2018;20(12):2869-2875.

40. Mifflin MD, St Jeor ST, Hill LA, Scott BJ, Daugherty SA, Koh YO. A new predictive equation for resting energy expenditure in healthy individuals. Am JClin Nutr. 1990;51(2):241-247.

41. Mittendorfer B, Horowitz JF, Klein S. Gender differences in lipid and glucose kinetics during short-term fasting. Am J Physiol Endocrinol Metab. 2001;281(6):E1333-E1339.

42. Allison DB, Paultre F, Maggio C, Mezzitis N, Pi-Sunyer FX. The use of areas under curves in diabetes research. Diabetes Care. 1995;18(2):245-250.

43. Van Cauter E, Mestrez F, Sturis J, Polonsky KS. Estimation of insulin secretion rates from C-peptide levels. Comparison of individual and standard kinetic parameters for C-peptide clearance. Diabetes. 1992;41(3):368-377.

44. Faul F, Erdfelder E, Lang AG, Buchner A. G*Power 3: a flexible statistical power analysis program for the social, behavioral, and biomedical sciences. Behav Res Methods. 2007;39(2):175-191. 